\title{
Neoadjuvant Intratumoral Cytokine-Loaded Microspheres are Superior to Postoperative Autologous Cellular Vaccines in Generating Systemic Anti-Tumor Immunity
}

\author{
ALISHA ARORA, MD, ${ }^{1}$ GANG SU, MD, ${ }^{1}$ EDITH MATHIOWITZ, PhD, ${ }^{2}$ JOSHUA REINEKE, ${ }^{2}$ \\ ALFRED E. CHANG, MD, ${ }^{1}$ AND MICHAEL S. SABEL, MD ${ }^{1 *}$ \\ ${ }^{1}$ Division of Surgical Oncology, University of Michigan, Ann Arbor, Michigan \\ ${ }^{2}$ Department of Molecular Pharmacology, Physiology and Biotechnology, Brown University, \\ Providence, Rhode Island
}

Background: Sustained intratumoral cytokine release using poly-lactic acid microspheres (PLAMs) can induce a systemic immune response, shifting immunotherapy to the neoadjuvant setting.

Methods: C57BL6 mice with established B16 melanomas underwent a single intralesional injection of IL-12, TNF- $\alpha$ or GM-CSF PLAM, alone or in combination. Tumor draining lymph nodes (TDLN) and spleens were assessed for a specific antitumor response by IFN $\gamma$ release assay and ELISPOT.

Results: Intralesional injection of TNF- $\alpha$, alone or in combination, resulted in significant tumor ablation. The induction of tumor specific reactive T-cells in the TDLN was greatest with IL-12 and TNF- $\alpha$. Only mice treated with IL-12 and TNF- $\alpha$ demonstrated a substantial T-cell response in cultured splenocytes. This combination resulted in a significant reduction in new tumors after re-challenge. Adjuvant therapy, using irradiated B16 cells in combination with equivalent doses of IL-12 and TNF- $\alpha$, failed to generate a similar T-cell response or prevent re-challenge.

Conclusions: Intratumoral IL-12 and TNF- $\alpha$ loaded PLAM leads to both local eradication of tumor and the induction of specific anti-tumor T-cells in the lymph nodes and spleens, resulting in memory immune response. Neoadjuvant treatment was significantly superior to postoperative autologous cellular vaccines using IL-12 and TNF- $\alpha$ PLAM.

J. Surg. Oncol. 2006;94:403-412. @ 2006 Wiley-Liss, Inc.

\footnotetext{
KeY Words: melanoma; microspheres; immunotherapy; intralesional; intratumoral; interleukin-12 (IL-12); tumor necrosis factor alpha (TNF- $\alpha$ ); granulocyte-macrophage colony stimulating factor (GM-CSF); in situ vaccination
}

\section{INTRODUCTION}

Activation of the immune system to produce an antitumor response depends on the presentation of antigen to T-cells by antigen presenting cells (APC) and the production of cytokines that promote a Th1 cytotoxic response. Various formulations of vaccines designed to activate a Th1 response using cytokines as adjuvant immunostimulants are presently being studied. These include the use of either dendritic cells exogenously loaded with tumor-associated antigens or autologous tumor cells that have been transfected with genes

(C) 2006 Wiley-Liss, Inc. encoding immunostimulatory cytokines and then delivered as a postoperative vaccine. Multiple cytokines have been investigated for this purpose with varying levels of success.

Grant sponsor: NIH; Grant number: CA102602-01; Grant sponsor: Walther Cancer Institute and Gillson-Longenbaugh Foundation.

*Correspondence to: Michael S. Sabel, MD, 3304 Cancer Center, 1500 East Medical Center Drive, Ann Arbor, MI 48109-0932. Fax: +734-647-9647. E-mail: msabel@umich.edu

Received 28 November 2005; Accepted 14 February 2006

DOI 10.1002/jso.20572

Published online in Wiley InterScience (www.interscience.wiley.com). 
Autologous cellular vaccines and dendritic cell vaccines are limited in both the amount of antigenic material that can be used and the levels and duration of cytokine release. An alternate approach is the sustained release of cytokines directly into tumors to provide not only local control, but also to stimulate a systemic immune response. This would shift the immunotherapy from an adjuvant setting to a neoadjuvant setting. We have recently described a novel method for delivering a local and sustained release of cytokines to the tumor microenvironment that does not require genetic manipulation [1]. Utilizing the B16 (murine melanoma) model, we sought to investigate the use of cytokine-loaded polylactic acid microspheres (PLAMs) as intralesional therapy for melanoma, to identify the optimum cytokines for therapy, and to delineate the most successful timing of therapy (neoadjuvant intratumoral therapy versus adjuvant vaccine therapy). We report here that the intralesional injection of the combination of IL-12 and TNF- $\alpha$ provides not only local control of the lesion, but generates a potent systemic anti-tumor immune response. This approach is superior to the use of a postoperative autologous cellular vaccine using these same cytokines as an adjuvant.

\section{MATERIALS AND METHODS}

\section{Mice and Tumors}

Six- to eight-week-old female C57B16 mice were purchased from The Jackson Laboratory (Bar Harbor, $\mathrm{NE}$ ) and maintained in specific pathogen-free conditions at the Animal Maintenance Facility of the University of Michigan Medical Center. B16-BL6 (B16) is a melanoma cell line of spontaneous origin that has been studied extensively [2]. MCA205 is a fibrosarcoma syngeneic to C57B16 mice induced by 3-methylcholanthrene and was used as a specificity control tumor [3]. Cell lines were maintained in complete media consisting of RPMI 1640 supplemented with $10 \%$ heat-activated fetal bovine serum, $0.1 \mathrm{mM}$ non-essential amino acids, $1 \mu \mathrm{M}$ sodium pyruvate, $100 \mu \mathrm{g} / \mathrm{ml}$ streptomycin, $100 \mathrm{units} / \mathrm{ml}$ penicillin, $50 \mu \mathrm{g} / \mathrm{ml}$ gentamycin and $0.5 \mu \mathrm{g} / \mathrm{ml}$ fungizone, all from Life Technologies, Inc. (Grand Island, NY). In vivo generation of tumors was accomplished by s.c. injection of C57B16 mice with $2 \times 10^{5}$ viable B16 cells. Tumors were measured every other day in two perpendicular dimensions ( $\mathrm{a}=$ length, $\mathrm{b}=$ width) with a Vernier caliper, and the size recorded as a volume $\left(\mathrm{mm}^{3}\right)$ as calculated by $a^{*} b^{2} / 2$. Statistical significance between groups was calculated using an unpaired Students $t$-test, with a $P$-value less than 0.05 considered statistically significant. Survival curves were generated using SPSS statistical software, version 13.0 (SPSS Inc., Chicago, IL). Statistical significance for disease-free survival was calculated using
Log-Rank analysis with a $P$-value less than 0.05 considered statistically significant.

\section{Microspheres}

A phase inversion nanoencapsulation technique was used for encapsulation of cytokines as described previously. Briefly, BSA (Sigma Chemical Co., St. Louis, MO), PLA $\left\{M_{r} 24,000\right.$ and $M_{r} 2,000$ (1:1, w/w): Birmingham Polymers, Inc., Birmingham, AL $\}$, and recombinant cytokine in methylene chloride (Fisher, Pittsburgh, PA) were rapidly poured into petroleum ether (Fisher) for formation of microspheres. Microspheres were filtered and lyophilized overnight for complete removal of solvent. Four formulations containing $1 \%$ BSA (w/w) were produced: (a) control (no cytokines); (b) murine IL-12 $\{\sim 5 \mu \mathrm{g} / \mathrm{mg}$ of PLA $\}$ (R\&D Systems, Minneapolis, MN); (c) murine GM-CSF $\{\sim 5 \mu \mathrm{g} / \mathrm{mg}$ of PLA \} (Biosource Intl, Camarillo, CA); (d) murine TNF- $\alpha$ $\{\sim 5 \mu \mathrm{g} / \mathrm{mg}$ of PLA $\}$ (R\&D Systems).

\section{Tumor-Draining LN Cells and Activation}

At varying time points after inoculation, TDLN and splenocytes were aseptically removed. Lymphoid cell suspensions were prepared by mechanical dissociation with 25-gauge needles and pressed with the blunt end of a 10 -ml plastic syringe in RPMI 1640. The cells were activated with $1 \mu \mathrm{g} / \mathrm{ml}$ anti-CD3 monoclonal antibody $(\mathrm{mAb})$ immobilized in 24 -well plates $\left(4 \times 10^{6}\right.$ cells $/ 2 \mathrm{ml} /$ well) for 2 days. The $\mathrm{LN}$ cells were subsequently cultured in recombinant human IL-2 (Chiron Therapeutics, Emeryville, CA) at $60 \mathrm{IU} / \mathrm{ml} \mathrm{IL}-2$ for 3 days at $2 \times$ $10^{5}$ cells $/ \mathrm{ml}$.

\section{Splenocytes and Activation}

Spleen cells obtained from the C57B16 mice were treated with ammonium chloride-potassium lysis buffer (0.83\% ammonium chloride, $0.1 \%$ KHCO3 and $0.004 \%$ EDTA) for $1 \mathrm{~min}$ to deplete erythrocytes and were washed twice with HBSS. Splenocytes were then activated with $1 \mu \mathrm{g} / \mathrm{ml}$ anti-CD3 mAb immobilized in 24 -well plates $\left(4 \times 10^{6}\right.$ cells $/ 2 \mathrm{ml} /$ well $)$ for 2 days. The $\mathrm{LN}$ cells were subsequently cultured in $60 \mathrm{IU} / \mathrm{ml} \mathrm{IL}-2$ for 3 days at $2 \times 10^{5}$ cells $/ \mathrm{ml}$.

\section{Measurement of In Vitro Cytokine Release}

$1 \times 10^{6}$ activated TDLN or splenocytes were cocultured with $1 \times 10^{6}$ B16 or MCA205 tumor cells irradiated to $15,000 \mathrm{cGy}$ in $2 \mathrm{ml}$ of $\mathrm{CM}$ per well of a 24-well tissue culture plate. IL-2 (4 IU/ml) was added at the beginning of the cultures, for $48 \mathrm{hr}$ at $37^{\circ} \mathrm{C}$. The supernatants were collected and, after centrifugation, analyzed for interferon- $\gamma$ (IFN $\gamma$ ) using commercially 
available ELISAs from PharMingen (San Diego, CA). A standard curve starting at $1,000 \mathrm{U} / \mathrm{ml}$ was established and 11 serial twofold dilutions were performed. Experimental values were computed with the use of regression analysis.

\section{ELISPOT Assay}

The number of IFN- $\gamma$ producing cells was measured using ELISPOT assay after intratumoral injection of cytokine-loaded PLAM. Briefly, 96-well plates were coated with anti-mouse IFN- $\gamma$ antibody (PharMingen). Activated splenocytes $\left(1 \times 10^{6}\right.$ cells/well $)$ were cultured for $48 \mathrm{hr}$ at $37^{\circ} \mathrm{C}$ in a $5 \% \mathrm{CO}_{2}$ incubator alone or in the presence of $4 \times 10^{5}$ irradiated B16 or MCA205 tumor cells. After that time, wells were washed and incubated overnight at $4{ }^{\circ} \mathrm{C}$ with a different clone of biotinylated anti-IFN- $\gamma$ antibody (PharMingen). Reactions were visualized and counted using anti-biotin-AP.

\section{RESULTS}

\section{Treatment of Established Tumors With an Intratumoral Injection of TNF- $\alpha$ Loaded PLAM Leads to Complete Regression of B16 Melanoma}

C57BL6 mice were inoculated subcutaneously with B16 melanoma in the right flank. Treatment groups comprised ten mice each. Six days after inoculation, when all mice had palpable subcutaneous tumors, mice were treated by a single intratumoral injection of cytokine-loaded microspheres at a dose of $2 \mathrm{mg}$ PLA/ tumor. Control mice were injected with a single injection of BSA-loaded microspheres. Treatment groups consisted of IL-12, GM-CSF or TNF- $\alpha$ either alone or in combination. Mice treated with combination cytokines were treated with $1 \mathrm{mg}$ PLA/tumor for each cytokine. Intratumoral injection of B16 tumors with PLAM loaded with IL-12, GM-CSF or the combination caused suppression of tumor growth, but did not lead to complete tumor regression (Fig. 1). B16 tumors treated with TNF$\alpha$, either alone or in combination with IL-12 or GM-CSF resulted in statistically significant tumor regression. Average tumor volume in mice treated with TNF- $\alpha$ alone or in combination was $19.35 \mathrm{~mm}^{3}$ compared to $163.6 \mathrm{~mm}^{3}$ for mice treated with IL-12, GM-CSF or the combination $(P<0.001$ by Students $t$-test $)$.

\section{Treatment of Established Tumors With an Intralesional Injection of Cytokine Loaded PLAM Induces Tumor-Specific Pre-effector Cells Both Regionally and Systemically}

C57BL6 mice with B16 tumors treated by intratumoral injection of PLAM loaded with IL-12, GM-CSF and

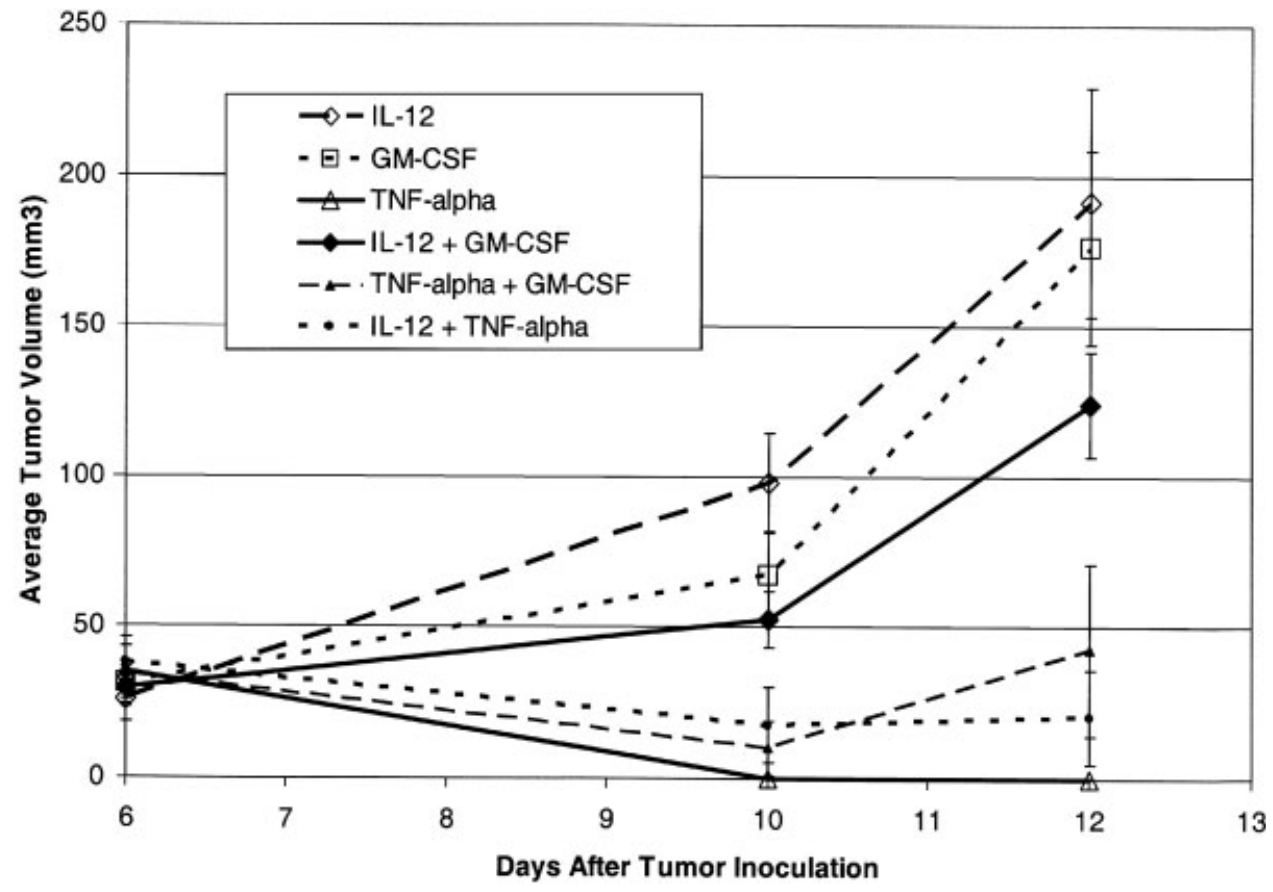

Fig. 1. Tumor volume after intratumoral injection of cytokine-loaded PLAM on primary tumor growth. C57B16 mice (five mice per group) were inoculated subcutaneously with $2 \times 10^{5}$ cells in the right flank. Palpable tumors were treated with a single intratumoral injection of poly-lacticacid microspheres encapsulating IL-12, TNF- $\alpha$, and GM-CSF, alone or in combination. Tumors were measured in two perpendicular dimensions $\left(\mathrm{a}=\right.$ length, $\mathrm{b}=$ width) and the size was recorded as a volume $\left(\mathrm{mm}^{3}\right)$ as calculated by $\mathrm{a} * \mathrm{~b} / 2$. By Day 6 after therapy, intratumoral injection of B16 tumors with TNF- $\alpha$ loaded PLAM, either alone or in combination with IL-12 or GM-CSF, resulted in statistically significant tumor regression $(P<0.001$ by Students $t$-test). 
TNF- $\alpha$, either alone or in combination, were sacrificed 7 days after treatment and tumor draining lymph nodes (TDLN) and splenocytes were harvested for anti-CD3/ IL-2 activation. Lymphocytes were then co-cultured either alone or in the presence of irradiated B16 cells and serum IFN- $\gamma$ levels were determined by ELISA. As a control for a tumor-specific response, activated lymphocytes were also co-cultured in the presence of MCA205, a sarcoma cell line. A tumor specific response is characterized as the difference in IFN $\gamma$ production between cells co-cultured with B16 versus MCA205. Intratumoral injection of IL-12 and TNF- $\alpha$ loaded PLAM resulted in the greatest B16-specific response in both the TDLN and splenocytes (Fig. 2). The ratio of the specific to non-specific tumor response is demonstrated in Figure 3.

To confirm these results, ELISPOT assay of the splenocytes was performed to quantify tumor-specific IFN- $\gamma$ producing cells. As with the IFN- $\gamma$ release assay, splenocytes from mice treated intralesionally with IL-12 and TNF- $\alpha$ demonstrated the greatest increase in B16 specific T-cells, compared to either IL-12 alone, TNF- $\alpha$ alone or GM-CSF alone or in combination. Mice treated with IL-12 and TNF- $\alpha$ had $75 / 10^{6} \pm 4.6$ IFN- $\gamma$ producing cells compared to $48.6 / 10^{6} \pm 2.6$ in the next highest group; TNF- $\alpha$ and GM-CSF $(P<0.01)$. The results are shown in Figure 4.

\section{Intratumoral Injection of IL-12 and TNF- $\alpha$ Loaded PLAM Prevents Re-Challenge After Treatment}

In a separate experiment, C57BL6 mice were inoculated subcutaneously with B16 melanoma in the right flank. Ten days after inoculation, when all mice had palpable subcutaneous tumors, mice were treated by a single intratumoral injection of IL-12 and TNF- $\alpha$ loaded microspheres either alone or in combination. Control mice were treated with microspheres containing BSA. Seven days after treatment, any residual tumor was surgically excised. One week after surgery, all mice were re-inoculated on the left flank with an identical tumorigenic dose of B16. Six of seven mice treated with BSA grew second tumors after treatment. As seen in Figure 5, intratumoral injection of either IL-12 or TNF- $\alpha$ alone did not prevent tumor growth when re-challenged. However, mice treated by the combination of IL-12 and TNF- $\alpha$ rejected tumors in 5 of 8 mice (Log Rank $\left.\chi^{2}=4.111, P=0.043\right)$.
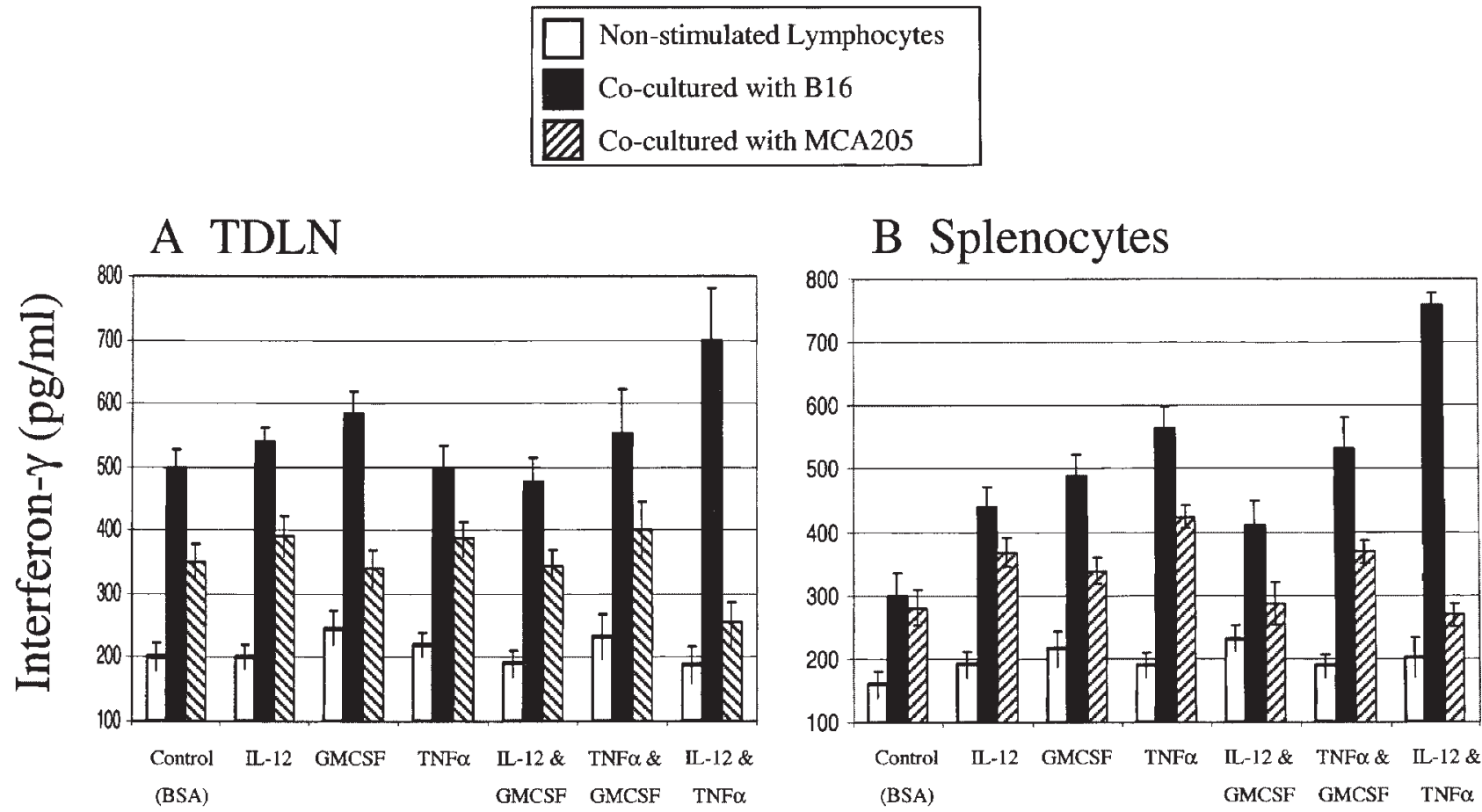

Fig. 2. Tumor specific IFN- $\gamma$ production by TDLN and splenocytes after intratumoral injection of cytokine-loaded PLAM. C57B16 mice (five mice per group) with B16 tumors were treated with a single intratumoral injection of poly-lactic-acid microspheres encapsulating IL-12, TNF- $\alpha$, and GM-CSF alone or in combination. One week later, TDLN and spleens were removed aseptically. Lymphoid single-cell suspensions were activated with anti-CD3 mAb and IL-2. Activated TDLN or splenocytes were cultured alone or with irradiated B16 or MCA205 tumor cells. The supernatants were collected and analyzed for IFN- $\gamma$ production. Data are derived from two individual experiments (each experiment included lymph nodes/spleens from 5 mice) (A) TDLN from mice treated with IL-12 and TNF- $\alpha$ PLAM initiated the greatest release of IFN- $\gamma$ in response to B16 tumor cells and not to MCA205 cells. B: Splenocytes from mice with B16 tumor treated with PLAM loaded with the combination of IL-12 and TNF- $\alpha$ also showed the greatest response to B16 tumor cells but not MCA205 cells. 


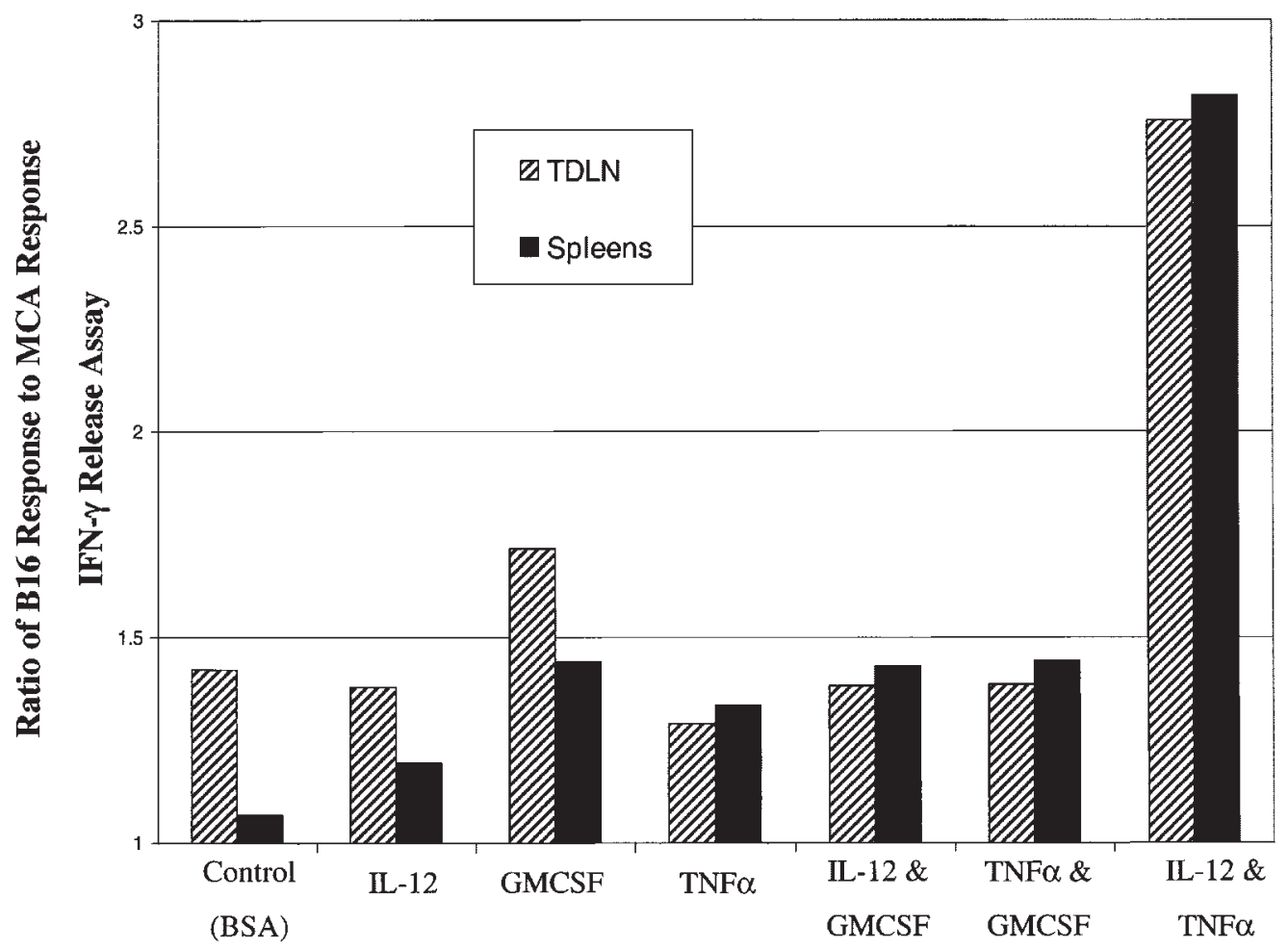

Fig. 3. Ratio of specific to non-specific IFN- $\gamma$ production by TDLN and splenocytes after intratumoral injection of cytokine-loaded PLAM.

\begin{tabular}{|l}
$\square$ Non-stimulated Lymphocytes \\
Co-cultured with B16 \\
Co-cultured with MCA205
\end{tabular}

\section{A Splenocytes}

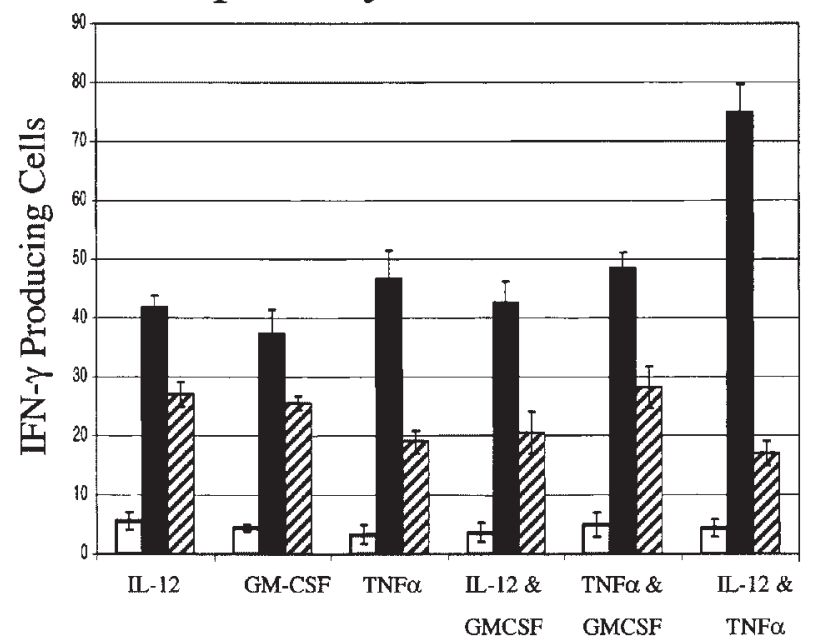

B Ratio of B16 to MCA Response

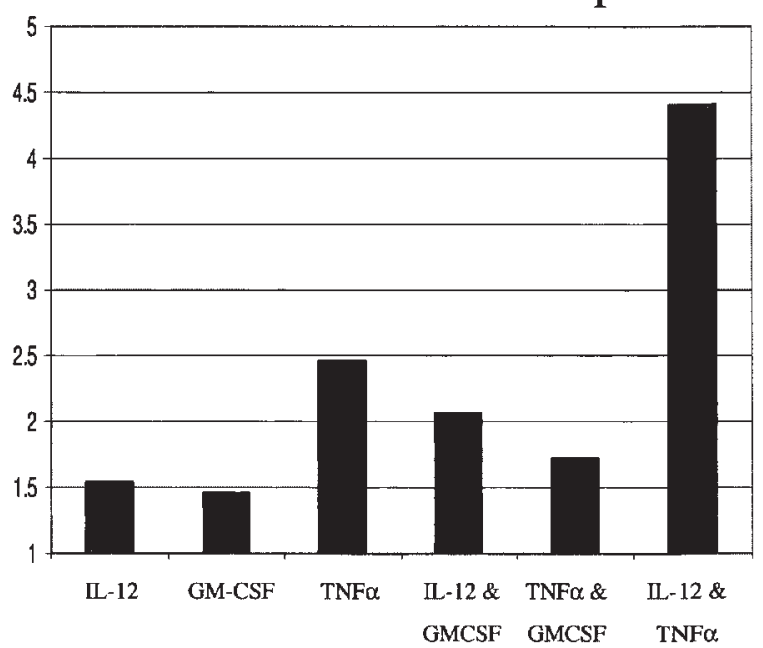

Fig. 4. IFN- $\gamma$ producing cells after intratumoral injection of cytokine-loaded PLAM as measured by ELISPOT assay. C57B16 mice (five mice per group) with B16 tumors were treated with a single intratumoral injection of poly-lactic-acid microspheres encapsulating IL-12, TNF- $\alpha$, and GM-CSF alone or in combination. One week later, spleens were removed aseptically. Lymphoid single-cell suspensions were activated with anti-CD3 mAb for 2 days and then cultured in $60 \mathrm{IU} / \mathrm{ml} \mathrm{IL-2} \mathrm{for} 3$ days. Activated splenocytes were co-cultured with irradiated B16 or MCA205 tumor cells. A: ELISPOT assay demonstrates that splenocytes from mice with B16 tumor treated with PLAM loaded with the combination of IL-12 and TNF- $\alpha$ also showed the greatest number of tumor-specific T-cells. $(P<0.01$ compared to each other group by $t$-test). B: Ratio of specific to non-specific IFN- $\gamma$ production by splenocytes after intratumoral injection of cytokine-loaded PLAM. 


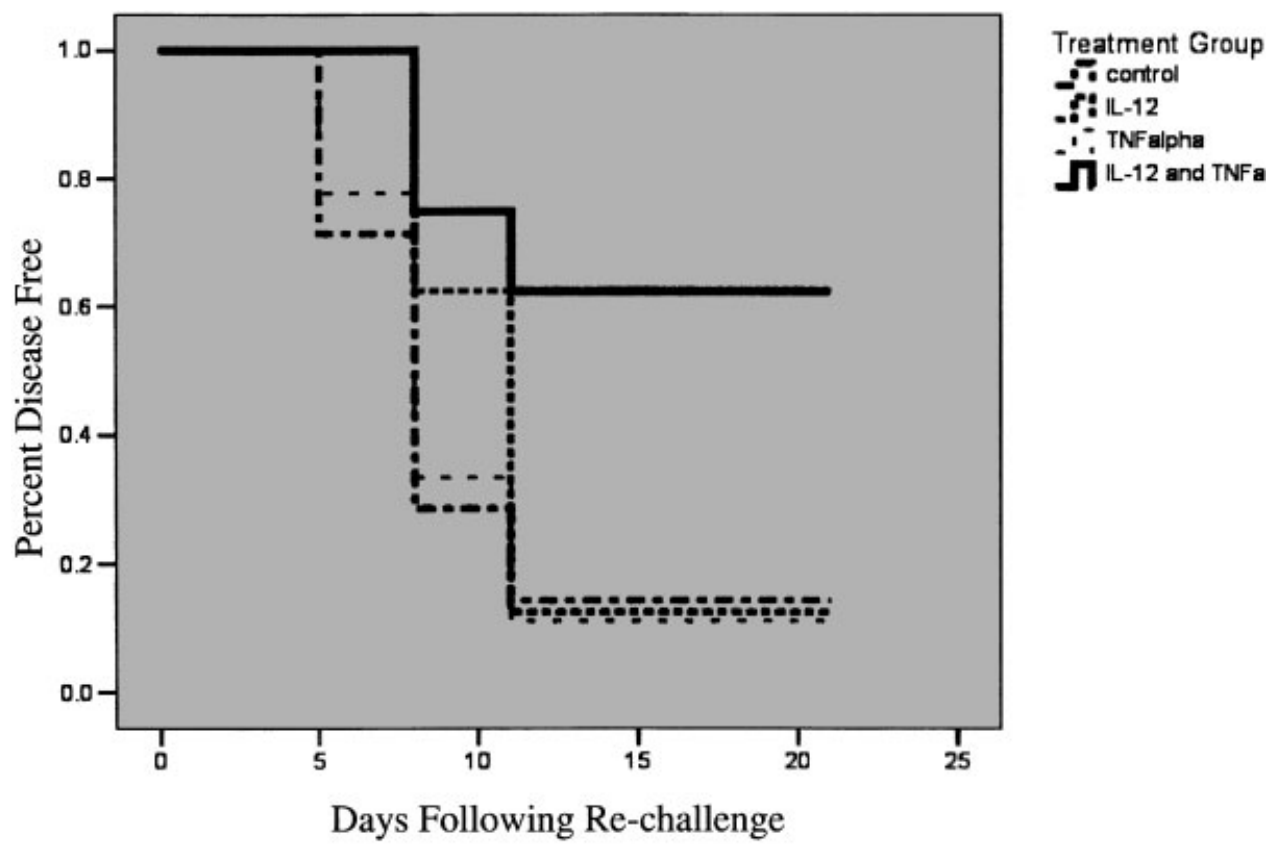

Fig. 5. Disease-free survival after re-challenge following intratumoral injection of cytokine-loaded PLAM and surgical excision. Mice treated by intratumoral injection of IL-12 and TNF- $\alpha$ loaded PLAM develop long-term immunity in comparison with the results of surgical excision alone. C57BL6 mice were inoculated with a subcutaneous injection of $2 \times 10^{5}$ B16 cells in the right flank. When all mice had palpable subcutaneous tumors, mice underwent injection with PLAM loaded with BSA controls, IL-12, TNF- $\alpha$ or IL-12 and TNF- $\alpha$. Seven days later, the residual tumor was resected and mice were then re-inoculated on the opposite flank with an identical tumorigenic dose of B16 cells. Treatment with IL-12 or TNF- $\alpha$ alone failed to inhibit the growth of tumors. The combination of IL-12 and TNF- $\alpha$, however, was able to significantly reduce the growth of tumors after re-challenge. (Log Rank $\left.\chi^{2}=4.111, P=0.043\right)$.

\section{Neoadjuvant Treatment of B16 Tumors With IL-12 and TNF- $\alpha$ PLAM Leads to a Greater Systemic Anti-Tumor Response Than an Adjuvant Vaccine}

C57BL6 mice were inoculated subcutaneously with B16 melanoma in the right flank. Eleven days after inoculation, all mice with palpable subcutaneous tumors underwent treatment. Mice in the neoadjuvant group were treated with either intratumoral injection of IL-12 or IL-12 and TNF- $\alpha$ loaded microspheres (2 mg PLAM/ mouse). Mice in the adjuvant group underwent surgical resection. One week later, the mice who had neoadjuvant treatment underwent surgical excision and the mice in the adjuvant group received vaccination in the form of a subcutaneous injection of $2 \times 10^{5}$ irradiated B16 cells in combination with IL-12 or IL-12 and TNF- $\alpha$ loaded microspheres. The dose of adjuvant cytokine was identical to the neoadjuvant group ( $2 \mathrm{mg}$ PLAM/mouse). One week later, all groups were sacrificed and TDLN and splenocytes were harvested (Fig. 6). Mice treated with neoadjuvant IL-12 and TNF- $\alpha$ had a significant, tumorspecific response demonstrable in both the TDLN and the splenocytes as compared to IL-12 alone in the neoadjuvant setting. IL-12 and TNF- $\alpha$ PLAM as adjuvants to a postoperative vaccine, however, failed to induce such a response.
To rule out whether the radiation altered the ability of the tumor cells to stimulate an immune response, naïve mice were vaccinated with live B16 cells in combination with IL-12, TNF- $\alpha$ or IL-12 and TNF- $\alpha$ loaded microspheres. Ten days after vaccination, TDLN and splenocytes were harvested and an IFN- $\gamma$ release assay was performed. IL-12 alone led to no increase in tumorspecific response compared to non-stimulated lymphocytes in either the TDLN (171.07 \pm 18.6 vs. $177.2 \pm$ $20.3)$ or splenocytes $(187.84 \pm 27.0$ vs. $167.71 \pm 15.4)$. TNF- $\alpha$ also had no significant effect. IL-12 and TNF- $\alpha$ led to only a mild increase in IFN $\gamma$ production (360.21 \pm 27.9 vs. $270.34 \pm 33.3$ in the TDLN, $345.43 \pm 35.0$ vs. $284.09 \pm 37.7$ in the splenocytes). Although the combination was superior to either cytokine alone, using live B16 cells was no better than irradiated cells in generating a significant tumor specific response in either the TDLN or splenocytes.

\section{Neoadjuvant Treatment of B16 Tumors With IL-12 and TNF- $\alpha$ PLAM is Superior to an Adjuvant Cellular Vaccine in Preventing Re-Challenge}

C57BL6 mice were inoculated subcutaneously with B16 melanoma in the right flank. Eleven days after inoculation, all mice with palpable subcutaneous tumors 

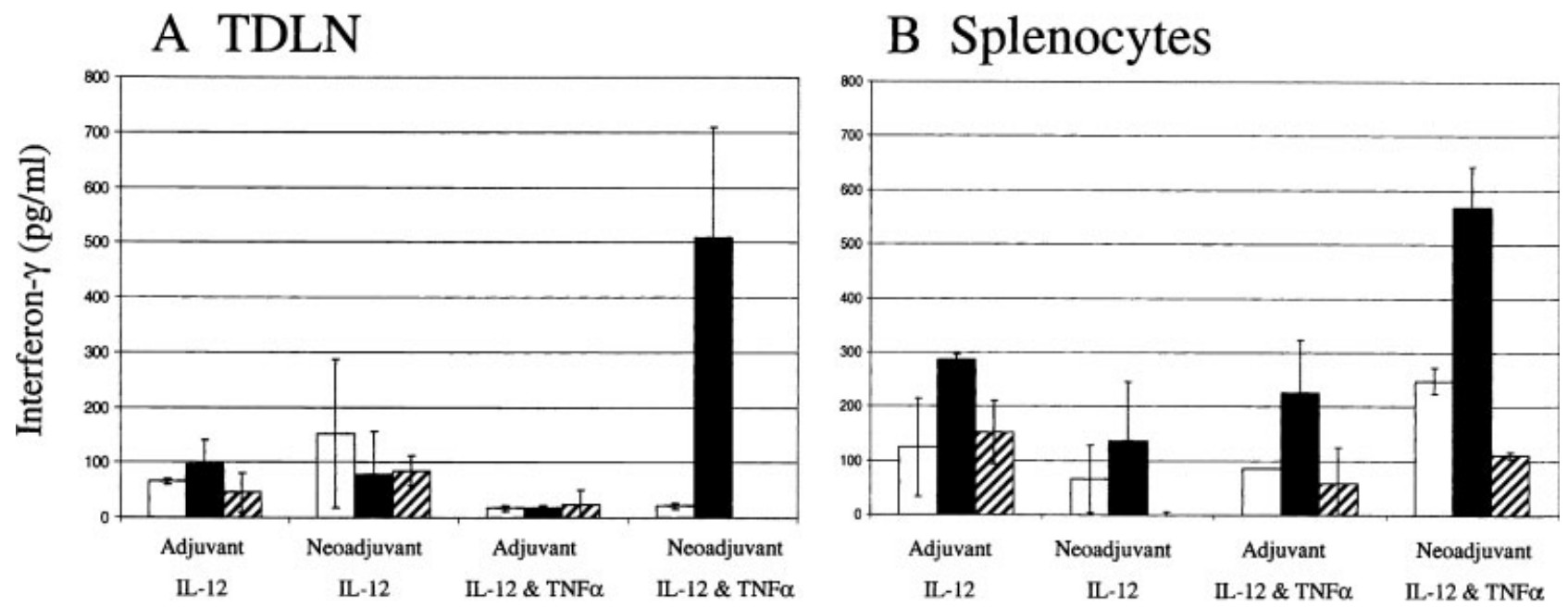

Fig. 6. Tumor-specific IFN- $\gamma$ production by TDLN and splenocytes after either neoadjuvant intratumoral treatment or postoperative autologous vaccination. C57B16 mice were inoculated with B16 melanoma and treated by one of two methods once subcutaneous tumors were present. Mice in the neoadjuvant group were treated with either intratumoral injection of IL-12 or IL-12 and TNF- $\alpha$ loaded microspheres ( $2 \mathrm{mg}$ PLAM/mouse). Mice in the adjuvant group underwent surgical resection. One week later, the mice who had neoadjuvant treatment underwent surgical excision and the mice in the adjuvant group received vaccination in the form of a subcutaneous injection of $2 \times 10^{5}$ irradiated B16 cells in combination with IL-12 or IL-12 and TNF- $\alpha$ loaded microspheres (at an identical dose to the neoadjuvant group). One week later, TDLN and spleens were removed aseptically. Lymphoid single-cell suspensions were activated with anti-CD3 mAb for 2 days and then cultured in $60 \mathrm{IU} / \mathrm{ml} \mathrm{IL}-2 \mathrm{for}$ 3 days. Activated TDLN (Fig. 5A) or splenocytes (Fig. 5B) were co-cultured with irradiated B16 or MCA tumor cells. The supernatants were collected and analyzed for IFN- $\gamma$ production. Only IL-12 and TNF- $\alpha$ in the neoadjuvant setting was able to generate a significant tumor-specific response $(P<0.01$ compared to each other group by Students $t$-test $)$.

underwent treatment. Mice in the neoadjuvant group were treated with IL-12 and TNF- $\alpha$ loaded microspheres (2 $\mathrm{mg} \mathrm{PLAM} / \mathrm{mouse}$ ). Mice in the adjuvant group underwent surgical resection. One week later, the mice who had neoadjuvant treatment underwent surgical excision and the mice in the adjuvant group received vaccination in the form of a dorsal cervical subcutaneous injection of $2 \times 10^{5}$ irradiated B16 cells in combination with IL-12 and TNF- $\alpha$ loaded microspheres (2 mg $\mathrm{PLAM} / \mathrm{mouse})$. One week later, all mice were rechallenged with subcutaneous injection in the left flank with a tumorigenic dose of B16 $\left(2 \times 10^{5}\right.$ cells $)$, and monitored for tumor growth. On Day 15 after rechallenge, 2 of $9(22 \%)$ mice treated with neoadjuvant IL-12 and TNF- $\alpha$ loaded PLAM prior to surgical resection developed tumors in the left flank, compared to 7 of 8 mice $(88 \%)$ treated with the adjuvant vaccine $\left(\log \operatorname{Rank} \chi^{2}=4.594, P=0.032\right.$; Fig. 7).

\section{CONCLUSIONS}

There has been significant interest in vaccination strategies that aim to develop immune responses specific to tumor-associated antigens, primarily the use of either autologous whole-cell vaccines or dendritic cells pulsed with autologous tumor lysate. The advantage of using autologous tumor is exposure to the large repertoire of tumor-associated antigens specific to the patient. The immunostimulatory effects of cytokines can be used to further improve the efficacy of these vaccines by recruiting and activating APC to the vaccination site, increasing the uploading and presentation of tumor antigens, and escalating the differentiation and proliferation of effector cells. As higher cytokine levels can be sustained at the site by local production, without the toxicity associated with systemic administration, autologous cellular vaccines and dendritic cells can be genetically modified with the gene of the cytokine of interest.

While cytokine gene-modified cellular and dendritic cell vaccines show the most potential from an immunologic standpoint, they are greatly hampered clinically by the availability of sufficient tumor. Patients with adequate harvestable tumor (those with bulky lymph node metastases or multiple metastatic foci), while possessing enough tumor to create a vaccine, have the worst 


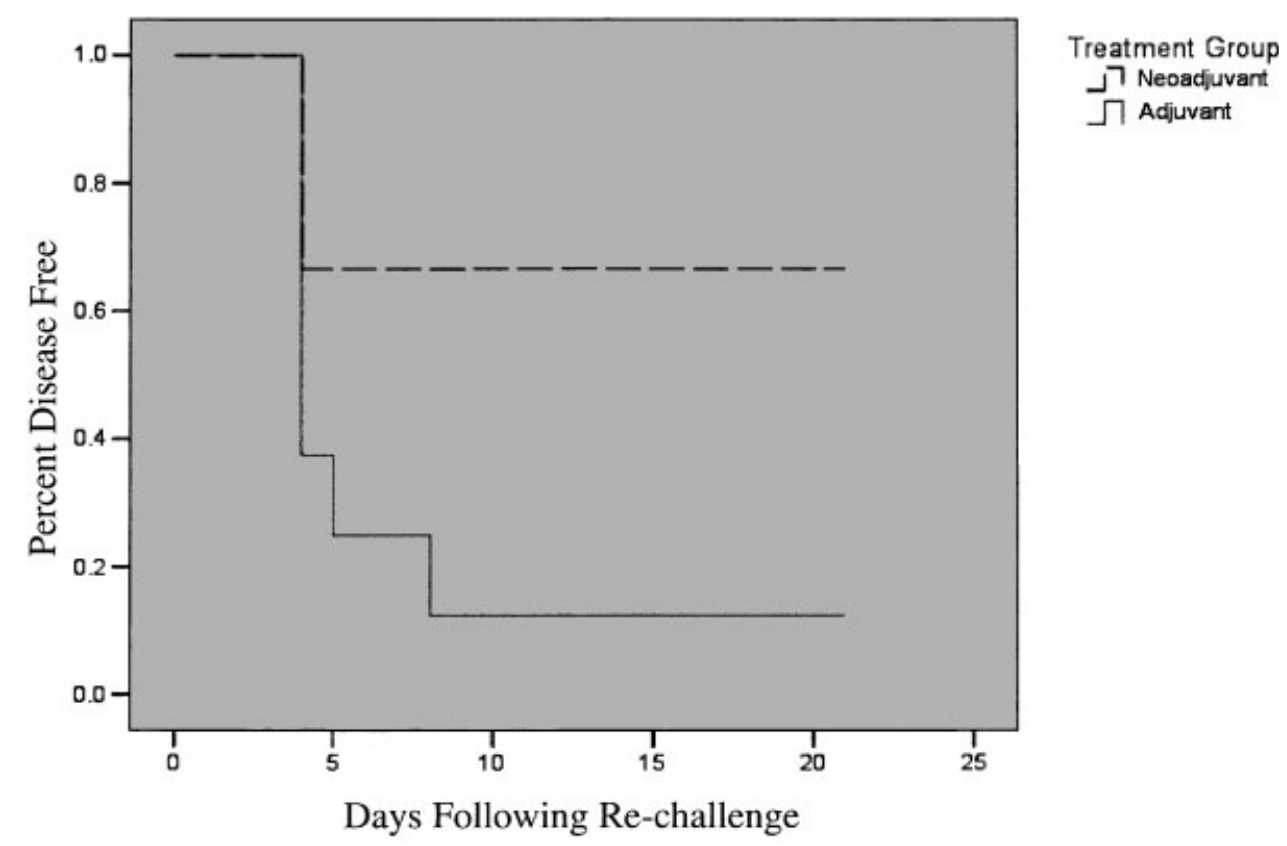

Fig. 7. Disease-free survival after re-challenge following intratumoral injection of cytokine-loaded PLAM and surgical excision. C57B16 mice were inoculated with B16 melanoma (five mice per group) and treated by one of two methods once subcutaneous tumors were present. Mice in the neoadjuvant group (- - - -) were treated with either intratumoral injection of IL-12 and TNF- $\alpha$ loaded microspheres ( 2 mg PLAM/mouse). Mice in the adjuvant group initially underwent surgical resection (- - . One week later, the mice who had neoadjuvant treatment underwent surgical excision and the mice in the adjuvant group received vaccination in the form of a subcutaneous injection of $2 \times 10^{5}$ irradiated B16 cells in combination with IL-12 and TNF- $\alpha$ loaded microspheres (at an identical dose to the neoadjuvant group). One week after this, mice were re-inoculated on the opposite flank with an identical tumorigenic dose of B16 cells. A postoperative autologous cellular vaccine, using IL-12 and TNF- $\alpha$ as adjuvant, failed to inhibit the growth of tumors. When delivered prior to surgical resection, however, this resulted in a significant resistance to re-challenge ( $\log$ rank $\left.\chi^{2}=4.594, P=0.032\right)$.

prognosis and are least likely to respond to therapy. Immunotherapy is most likely to be effective in the adjuvant setting, but these patients rarely have an extremely limited availability of tumor. Therefore, new approaches to immunotherapy are needed, those that utilize autologous whole cells but do not require ex vivo manipulation. One such approach is in situ vaccination, which consists of delivering cytokines directly to the site of the tumor [4-11]. In this way, the tumor itself serves as the vaccine, precluding the cost and difficulty in establishing a cell line and transfecting it with the gene for a cytokine. We have previously described an alternate strategy for in situ cytokine delivery: the use of slowrelease polymer microspheres $[1,12,13]$. Using the poorly immunogenic B16 (murine melanoma) model, we sought to identify the optimum cytokines (or combination) for in situ vaccination, and to compare this neoadjuvant approach to the adjuvant use of autologous cellular vaccines.

The result confirms the synergistic effects of intratumoral IL-12 and TNF- $\alpha$ in generating a systemic antitumor response. In this model, neither cytokine alone nor GM-CSF alone or their combination was effective. IL-12 exerts potent Th1-inducing effects and augments NK cell cytotoxicity [15,16]. IL-12 activates and mobilizes tumor-alerted NK and T-cells in lymphoid organs, and seems to exert its greatest effect in those sites where Tlymphocytes have been activated and have accumulated $[17,18]$. Against a poorly immunogenic tumor such as B16 melanoma, intratumoral IL-12 alone may not be able to stimulate enough inflammation and tumor necrosis to initiate the activation of T-cells. Combining IL-12 with other agents may therefore enhance its anti-tumor efficacy. Previous reports have demonstrated increased activity when IL-12 is combined with GM-CSF [19], IL$18[6,20]$, and IL-2 [10,21]. TNF- $\alpha$ is a multipotent cytokine that causes hemorrhagic necrosis in tumors, evokes apoptosis and activates immune cells [22]. As with IL-12, while some tumor cell lines are killed by TNF- $\alpha$ alone, others are not at all affected, and require the combination of TNF- $\alpha$ with other cytokines [23]. Our results confirm that the combination of IL-12 and TNF- $\alpha$ appears to be a particularly effective combination. Synergistic effects possibly include skewing towards a Th1 response [24], induction of the tumoricidal activity of macrophages [25], and increased necrosis and tumor infiltration of both PMN and CD8+ cells at the site of the tumor [13].

Given the synergy seen between IL-12 and TNF- $\alpha$, we sought to examine whether the cytokines are better used 
intratumorally as opposed to adjuvants to postoperative vaccines. The use of intratumoral therapy would shift the timing of immunotherapy from postoperative to preoperative. These results clearly demonstrate that neoadjuvant therapy is superior to adjuvant therapy in both stimulating an anti-tumor T-cell response and preventing tumor growth when re-challenged. Postoperative vaccines are limited by both the amount of tumor (and tumor-associated antigens) available for uptake and the need to irradiate the tumor cells, which has been shown to induce less of an immune response, perhaps contributing to the ineffectiveness of postoperative vaccines [26]. However, using live tumor cells for vaccination in combination with IL-12 and TNF- $\alpha$, failed to generate results similar to intratumoral therapy, suggesting that the irradiation was not the reason why the vaccine failed.

This makes a strong argument that in situ vaccination may be a better immunologic approach than postoperative vaccination. In addition, there are other potential advantages to the neoadjuvant approach. In situ vaccination precludes the need to harvest adequate amounts of tumor or manipulate these cells ex vivo, which is not only time consuming and cost-prohibitive, but not technically feasible for many solid tumors. Chemotherapy given prior to surgical resection of a solid tumor can offer an increased likelihood of complete resection and may increase the odds of cure by reducing or eliminating micrometastatic disease at an earlier time point. This same premise may be applied to immunotherapy as well.

In conclusion, intratumoral IL-12 and TNF- $\alpha$ loaded PLAMs results in not only tumor ablation, but also in the generation of a regional and systemic tumor-specific immune response capable of preventing re-challenge and improving disease-free survival after resection. The local and sustained release of these cytokines in the neoadjuvant setting is greatly superior to their use as an adjuvant to a postoperative autologous cellular vaccine. Based on these results, we are now examining the mechanisms behind the apparent synergistic effects between IL-12 and TNF- $\alpha$ at both the site of injection in the primary tumor and on established metastatic disease. This information will hopefully further guide the clinical applicability of these cytokines in the neoadjuvant setting and suggest additional methods to augment the resultant anti-tumor immune response. Furthermore, these results suggest there may be a clinical benefit to shifting away from postoperative adjuvant immunotherapy towards preoperative intratumoral immunotherapy.

\section{ACKNOWLEDGMENTS}

This work was in part funded by NIH Grant CA102602-01 and grants from the Walther Cancer Institute and Gillson-Longenbaugh Foundation.

\section{REFERENCES}

1. Egilmez NK, Jong YS, Sabel MS, et al.: In situ tumor vaccination with interleukin-12-encapsulated biodegradable microspheres: Induction of tumor regression and potent antitumor immunity. Cancer Res 2000;60:3832-3837.

2. Hart IR: The selection and characterization of an invasive variant of the B16 melanoma. Am J Pathol 1979;97:587-600.

3. Barth RJ, Bock SN, Mule' JJ, et al.: Unique murine tumorassociated antigens identified by tumor infiltrating lymphocytes. J Immunol 1990;144:1531-1537.

4. Crittenden MR, Thanarajasingam U, Vile RG, et al.: Intratumoral immunotherapy: Using the tumour against itself. Immunology 2005;114:11-22.

5. Sharma S, Yang SC, Batra RK, et al.: Intratumoral therapy with cytokine gene-modified dendritic cells in murine lung cancer models. Methods Mol Med 2003;75:711-722.

6. Tatsumi T, Huang J, Gooding WE, et al.: Intratumoral delivery of dendritic cells engineered to secrete both interleukin (IL)-12 and IL-18 effectively treats local and distant disease in association with broadly reactive Tc1-type immunity. Cancer Res 2003;63: 6378-6386.

7. Pan PY, Li Y, Li Q, et al.: In situ recruitment of antigen-presenting cells by intratumoral GM-CSF gene delivery. Cancer Immunol Immunother 2004;53:17-25.

8. Lucas ML, Heller R: IL-12 gene therapy using an electrically mediated nonviral approach reduces metastatic growth of melanoma. DNA Cell Biol 2003;22:755-763.

9. Shi F-S, Rakhmilevich AL, Heise CP, et al.: Intratumoral injection of interleukin-12 plasmid DNA, either naked or in complex with cationic lipid, results in similar tumor gregression in a murine model. Mol Cancer Ther 2002;1:949-957.

10. Addison CL, Bramson JL, Hitt MM, et al:: Intratumoral coinjection of adenoviral vectors expressing IL-2 and IL-12 results in ehanced frequency of regression of injected and untreated distal tumors. Gene Ther 1998;5:1400-1409.

11. Tahara H, Zitvogel L, Storkus WJ, et al.: Effective eradication of established murine tumors with IL-12 gene therapy using a polycistronic retroviral vector. J Immunol 1995;154:6466-6474.

12. Sabel MS, Hill H, Jong YS, et al.: Neoadjuvant therapy with interleukin-12-loaded polylactic acid microspheres reduces local recurrence and distant metastases. Surgery 2001;130:470-478.

13. Sabel MS, Skitzki J, Stoolman L, et al.: Intratumoral IL-12 and TNF-alpha-loaded microspheres lead to regression of breast cancer and systemic antitumor immunity. Ann Surg Oncol 2004; 11:147-156.

14. Rook AH, Wood GS, Yoo EK, et al.: Interleukin-12 therapy of cutaneous T-cell lymphoma induces lesion regression and cytotoxic T-cell responses. Blood 1999;94:902-908.

15. Trinchieri G: Interleukin-12: A cytokine at the interface of inflamation and immunity. Adv Immunol 1998;70:83-243.

16. Lamont AG, Adorini L: IL-12: A key cytokine in immune regulation. Immunol Today 1996;17:214-217.

17. Cavallo F, Signorelli P, Giovarelli M, et al.: Antitumor efficacy of adenocarcinoma cells engineered to produce Interleukin 12 (IL12) or other cytokines compared with exogenous IL-12. JNCI 1997;89:1049-1058.

18. Trembleau S, Germann T, Gately MK, et al.: The role of IL-12 in the induction of organ-specific autoimmune diseases. Immunol Today 1995;16:383-386.

19. Hill HC, Conway TF, Jr., Sabel MS, et al.: Cancer immunotherapy with interleukin 12 and granulocyte-macrophage colony-stimulating factor-encapsulated microspheres: Coinduction of innate and adaptive antitumor immunity and cure of disseminated disease. Cancer Res 2002;62:7254-7263.

20. Tamura T, Nishi T, Goto T, et al.: Combination of IL-12 and IL-18 of electro-gene therapy synergistically inhibits tumor growth. Anticancer Res 2003;23:1173-1179.

21. Wigginton JM, Komschiles KL, Back TC, et al.: Administration of interleukin-12 with pulse interleukin-2 and the rapid and complete eradication of murine renal carcinoma. JNCI 1996; 88:38-43. 
22. Meuller H: Tumor necrosis factor as an antineoplastic agent Pitfalls and promises. Cell Mol Life Sci 1998;54:1291-1298.

23. Sugarman BJ, Aggarwal BB, Hass PE, et al.: Recombinant human tumor necrosis factor-a: Effects on proliferation of normal and transformed cells in vitro. Science 1998;230:943-945.

24. Ahlers JD, Belyakov IM, Matsui S, et al.: Mechanisms of cytokine synergy essential for vaccine protection against viral challenge. Int Immunol 2001;13:897-908.
25. Hori K, Ehrke MJ, Mace K, et al.: Effect of recobminant tumor necrosis factor on tumoricidal activation of murine macrophages: Synergism between tumor necrosis factor and gamma-interferon. Cancer Res 1987;47:5868-5874.

26. Tai KF, Chen DS, Hwang LH: Curative potential of GM-CSF secreting tumor cells vaccines on established orthotopic liver tumors: Mechanisms for the superior antitumor activity of life tumor cell vaccines. J Biomed Sci 2004;11:228-238. 